International Journal of Medical Sciences

ISSN 1449-1907 www.medsci.org 2008 5(5):292-294

Short Research Communication

(C) Ivyspring International Publisher. All rights reserved

\title{
Simultaneous dislocation of the radial head and distal radio-ulnar joint. A case report
}

\author{
D-A.J. Verettas, G.I. Drosos, K.C. Xarchas, C.N. Chatzipapas ${ }^{\bowtie}$, C. Staikos
}

Department of Orthopaedic Surgery, Medical School, Democritus University of Thrace, University General Hospital of Alexandroupolis, 68100 Alexandroupolis, Greece.

$\triangle$ Correspondence to: Christos N. Chatzipapas, MD, PhD, Orthopaedic Surgeon. Department of Orthopaedic Surgery, Medical School, Democritus University of Thrace, University General Hospital of Alexandroupolis, 68100 Alexandroupolis, Greece. Tel. +302551036550 Fax. +302551023326 Email hatzy@otenet.gr

Received: 2008.09.04; Accepted: 2008.09.23; Published: 2008.09.29

Isolated dislocation of the distal radio-ulnar joint and isolated dislocation of the radial head in adults are not common injuries. A simultaneous dislocation of the radial head and distal radio-ulnar joint with no other injury seems to be extremely rare since only one report was found in the English literature. A similar case, but with some differences in presentation and treatment is reported.

Key words: Simultaneous; Dislocation; Distal radio-ulnar joint; Radial head

\section{Introduction}

Posttraumatic instability of the distal radio-ulnar joint (DRUJ) has been commonly described in association with Colles's type fractures [1], Smith's type fractures [2], fractures of the radial diaphysis (Galeazzi's fracture-dislocation) [2], fractures of the radial head [3] and elbow dislocations $[4,5]$.

Isolated dislocation of the DRUJ, although it has been described since 1777 by Desault [6], only few cases have been reported according to a recent review [7]. Isolated dislocation of the radial head is common in children but rare in adults as only 20 cases have been reported during the last 30 years [8].

A simultaneous dislocation of the radial head and distal radio-ulnar joint with no other injury seems to be extremely rare since only one report was found in the English literature [7].

\section{Case report}

A 54-year-old male presented in the Emergency Department of our Institution, complaining of pain, deformity of the left wrist and elbow and inability to move these joints, following a fall on the out-stretched hand at work. On clinical examination, the forearm was held in supination with both the elbow and the wrist in flexion. He was unable to move the elbow and the wrist, whilst finger movement and sensation were normal. No neurovascular injury could be detected. No other parts of the body were injured.

Radiographs of the forearm, including both the elbow and wrist joints, revealed an anterior dislocation of the radial head and a disruption of the DRUJ with a volar dislocation of the distal ulna [Fig.1].

The injury was treated immediately, with closed reduction of both dislocations, K-wire stabilization of the DRUJ and immobilization of the forearm with a long arm cast in supination. Under general anaesthesia firstly the radial head was reduced with traction, supination and direct pressure, followed by reduction of the DRUJ with pronation, while the reduced radial head was maintained in supination by an assistant. After the reduction, the radial head was unstable whenever the forearm was positioned in neutral rotation. Therefore it was decided to stabilise the DRUJ with a K-wire in the reduced position and immobilise the forearm with a long arm cast in supination and the elbow in 90 degrees flexion [Fig.2].

Three weeks later the elbow was allowed to be mobilised while the DRUJ K-wire remained in situ for a total of 12 weeks. Three months later the patient had regained full range of elbow movement but with some restriction in forearm pronation, with a stable DRUJ. Unfortunately the patient did not attend any further appointments in the Outpatient Department. 

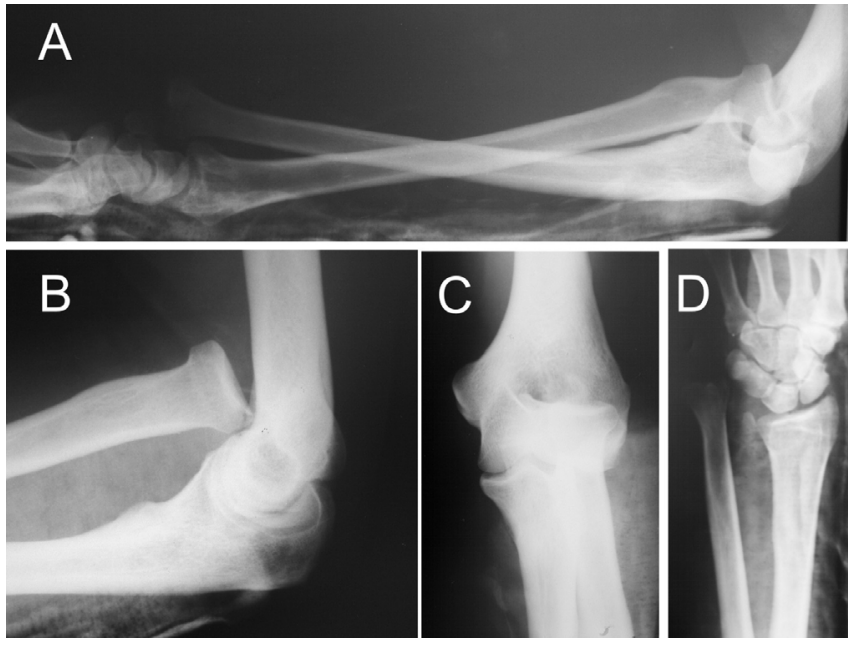

Figure 1. Initial radiographs showing the anterion dislocation of the radial head (A, B, C) and the volar dislocation of the distal radio-ulnar joint $(\mathrm{A}, \mathrm{D})$.

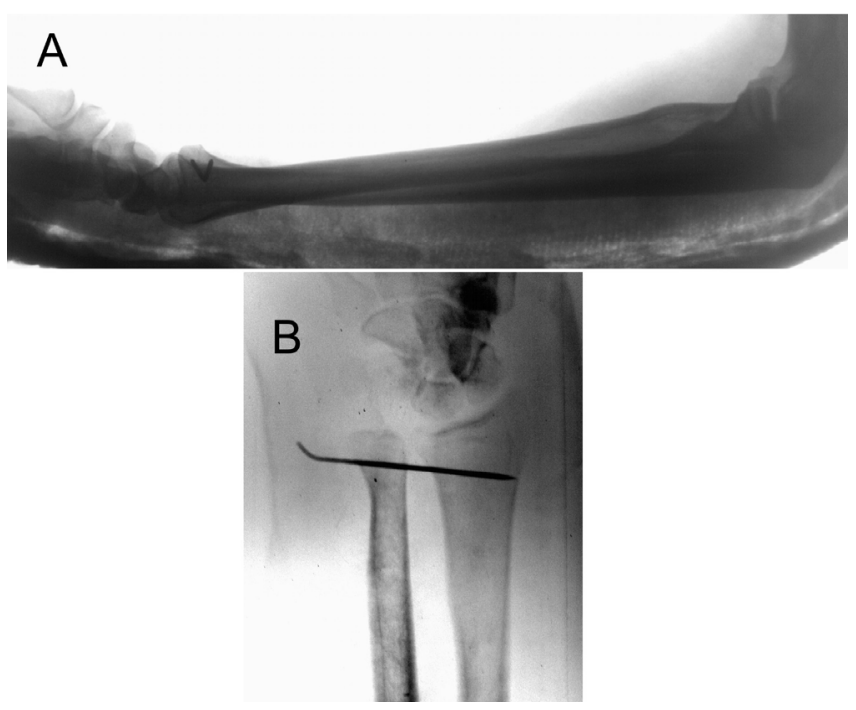

Figure 2. Post-operative lateral radiograph of the forearm (A) and anterior-posterior radiograph of the wrist (B).

\section{Discussion}

A similar case with a simultaneous dislocation of the radial head and DRUJ with no other injury of the arm was reported a few years ago [7]. The differences with our case is that (a) in our case the radial head was displaced purely anteriorly and not antero-lateraly as in their case and (b) after the reduction, in our case the radial head was unstable with the forearm in neutral rotation.
Dislocations of the DRUJ are classified according to the displacement of the ulna as dorsal (more common), volar and longitudinal (proximal translation), the latter being the original Essex-Lopresti injury [3,9]. The mechanism of injury for the dorsal dislocation is hyperpronation, for the volar dislocation hypersupination and for the original Essex-Lopresti injury is the proximal translation of the radius [3,9].

Isolated radial head dislocation may be posterior (dorsal) or anterior (volar) and the mechanism is hyperpronation and hypersupination of the forearm respectively $[8,10]$.

As far as the mechanism of injury of the simultaneous dislocation of both joints is concerned we agree with the mechanism proposed by Leung et al [7]. They named this injury 'criss-cross' injury of the forearm suggesting that the interosseous membrane may play a role and function as a pivot between the two forearm bones.

The same authors also suggested that, according to their cadaveric studies, this combined injury may represent a severe form of isolated dislocation of one of the radio-ulnar joints. They believe that even in an isolated dislocation of one of the radio-ulnar joints, a subluxation or a sprain of the other joint is present. Therefore the dislocation of the other joint is just a subsequent stage of the same injury.

Although an increase in the distance at the DRUJ was observed on AP view (figure 2), the reduction was accepted. In fact, a perfect reduction was not achieved. It would be interesting to see if this would have caused problems in the patient's wrist after some period of time. Unfortunately we were able to follow-up the patient only for three months post-operatively.

\section{Conflict of Interest}

The authors have declared that no conflict of interest exists.

\section{References}

1. Colles A. On the fracture of the carpal extremity of the radius. Edinburgh Med Surg J. 1814; 10: 182-86.

2. Hyman G, Martin FRR. Dislocation of the inferior radio-ulnar joint as a complication of fracture of the radius. Br J Surg. 1940; 27: 481-91.

3. Essex-Lopresti P. Fractures of the radial head with distal radio-ulnar dislocation: report of two cases. J Bone Joint Surg [Br]. 1951; 33: 244-47.

4. Bock GW, Cohen MS, Resnick D. Fracture-dislocation of the elbow with inferior radioulnar dislocation: a variant of the Essex-Lopresti injury. Skeletal Radiol. 1992; 21: 315-17.

5. Spicer DD, Hargreaves D, Eckersley R. Simultaneous Dislocations of the Radiocapitellar and Distal Radioulnar Joints. J Orthop Trauma. 2002; 16: 136-38.

6. Cotton FJ, Brickley WJ. Luxation of the ulna forward at the wrist (without fracture). Ann Surg. 1912; 55: 368-73.

7. Leung YF, Ip SPS, Wong A, Wong KN, Wai YL. Isolated dislocation of the radial head, with simultaneous dislocation of proxi- 
mal and distal radio-ulnar joints without fracture in an adult patient: case report and review of the literature. Injury. 2002; 33: 271-73.

8. Rethnam U, Yesupalan RSU, Bastawrous SS. Isolated radial head dislocation, a rare and easily missed injury in the presence of major distracting injuries: a case report. J Med Case Reports. 2007; $1: 38$.

9. Alexander AH. Bilateral traumatic dislocation of the distal radioulnar joint, ulna dorsal: case report and review of the literature. Clin Orthop Rel Res. 1977; 129: 238-44.

10. Deehan DJ, Ashcroft GP, Hutchison JD. Isolated dislocation of the radial head in an adult: case report and review of the literature. J Roy College Surgeons Edinburgh. 1995; 40: 421-23. 